\title{
The Chandra survey of Carina OB stars
}

\author{
Marc Gagné ${ }^{1}$, Garrett Fehon ${ }^{1}$, Michael R. Savoy ${ }^{1}$, David H. Cohen ${ }^{2}$, \\ Leisa K. Townsley ${ }^{3}$, Patrick S. Broos ${ }^{3}$, Matthew S. Povich ${ }^{3}$, \\ Michael F. Corcoran ${ }^{4}$, Nolan R. Walborn ${ }^{5}$, Anthony F.J. Moffat ${ }^{6}$, \\ Yael Nazé ${ }^{7}$, Lidia M. Oskinova ${ }^{8}$ \\ ${ }^{1}$ Dept. of Geology and Astronomy, West Chester Univ., West Chester, PA 19383 \\ ${ }^{2}$ Dept. of Physics and Astronomy, Swarthmore College, Swarthmore, PA 19081 \\ ${ }^{3}$ Dept. of Astronomy \& Astrophysics, Penn State Univ., University Park, PA 16802 \\ ${ }^{4}$ CRESST and X-ray Astrophysics Laboratory, NASA/GSFC, Greenbelt, MD 20771 \\ ${ }^{5}$ Space Telescope Science Institute, Baltimore, MD 21218, USA \\ ${ }^{6}$ Dépt. de Physique, Univ. de Montréal, Succ. Centre-Ville, Montréal, QC, H3C 3J7, Canada \\ ${ }^{7}$ GAPHE, Dépt. AGO, Univ. Liège, Allée du 6 Août 17, Bat. B5C, B4000-Liège, Belgium \\ ${ }^{8}$ Institute for Physics and Astronomy, Univ. of Potsdam, 14476 Potsdam, Germany
}

\begin{abstract}
We have combined 22 deep Chandra ACIS-I pointings to map over one square degree of the Carina complex. Our x-ray survey detects 69 of 70 known O-type stars and 61 of 130 known early $\mathrm{B}$ stars. The majority of single $\mathrm{O}$ stars display soft X-ray spectra and have a mean $\log L_{\mathrm{X}} / L_{\mathrm{bol}} \approx-7.5$ suggesting shocks embedded in the O-star winds. Over OB stars show unusually high X-ray luminosities, high shock temperatures or time variability, not predicted for embedded wind shocks.
\end{abstract}

Keywords. X-rays: stars, X-rays: binaries, stars: early-type, stars: pre-main-sequence

The Chandra Carina Complex Project (CCCP) survey area contains over 200 known massive stars: the LBV $\eta$ Car, the Wolf Rayet stars WR 22, WR 24 and WR 25, 70 known O stars, and 130 B0-B3 stars with known spectral types. We have constructed a searchable electronic database of x-ray and optical properties for the 200 Carina OB stars. We divide the massive star population in Carina into four main groups based on spectral type and luminosity class: LBV/WR stars (4), O-type binaries (15), O-type single stars (55), and B0-B3 stars (130). We note that none of the B stars are typed as spectroscopic binaries. The goal of this study is to characterize $L_{\mathrm{X}}, L_{\mathrm{X}} / L_{\mathrm{bol}}$, the X-ray temperatures $k T$, and the temporal variability of single and binary OB stars, to look for new candidate colliding wind systems (e.g., Stevens et al. 1992) and magnetically confined wind shock sources (e.g., ud-Doula \& Owocki 2002).

The Chandra CCD spectra of the 78 OB stars with more than 50 ACIS counts were fit in XSPEC using a one- or two-temperature APEC emission model, and a two-component absorption model: a fixed TBABS column density to represent cold, neutral ISM absorption (assuming $A_{V} / N_{\mathrm{H}}=1.6 \times 10^{21} \mathrm{~cm}^{-2}$ per mag), and free column density to represent absorption from the overlying stellar wind. $L_{\mathrm{X}}$ was calculated correcting only for the ISM column. For single $\mathrm{O}$ stars, $\log L_{\mathrm{X}} / L_{\mathrm{bol}}=-7.59 \pm 0.23$, with no such trend for B stars.

Table 1 highlights three notable single O stars: HD 93250, O4 III(fc), MJ 496, O8.5 V, and MJ 449, O8.5 V((f)), all of which have $k T>1.7 \mathrm{keV}, \log L_{\mathrm{X}} / L_{\mathrm{bol}}>-7.1$, and two of which are time variable. These three stars do not have massive, spectroscopic companions, and their $L_{\mathrm{X}}$ is too high to be produced by unseen, lower-mass pre-mainsequence (PMS) companions. For these three we consider two hypotheses: a more distant massive companion (as is the case for HD 93129A), or magnetically confined wind shocks (as in $\theta^{1}$ Ori C, Gagné et al. 2005). 
Table 1. Notable stars: XSPEC and time variability parameters

\begin{tabular}{llllllc}
\hline Star name & ACIS name & Spectral type & $\log L_{\mathrm{X}}\left(\mathrm{erg} \mathrm{s}^{-1}\right)$ & $\log \frac{L_{\mathrm{X}}}{L_{\mathrm{bol}}}$ & $k T_{\mathrm{avg}}(\mathrm{keV})$ & $P_{\mathrm{KS}}(\%)$ \\
\hline HD 93250 & $104445.04-593354.6$ & O4 III(fc) & 33.12 & -6.41 & 2.30 & 20 \\
MJ 496 & $104508.23-594607.0$ & O8.5 V & 32.09 & -6.46 & 1.70 & 14 \\
MJ 449 & $104454.70-595601.8$ & O8.5 V(f)) & 31.24 & -7.08 & 3.09 & 6 \\
\hline HD 93403 & $104544.13-592428.1$ & O5.5 I + O7 V & 32.93 & -6.60 & 1.00 & 0 \\
HD 93205 & $104433.74-594415.4$ & O3 V + O8 V & 32.55 & -6.82 & 0.30 & 3 \\
HD 93129A & $104357.47-593251.3$ & O2 If* & 32.91 & -6.85 & 0.74 & 46 \\
HD 93343 & $104512.23-594500.5$ & O8 V + O7-8.5 V & 31.45 & -7.19 & 3.17 & 30 \\
QZ Car & $104422.91-595935.9$ & O9.7 I + O8 III & 32.55 & -7.26 & 1.03 & 5 \\
FO 15 & $104536.33-594823.5$ & O5.5 Vz + O9.5 V & 30.62 & -8.26 & 0.50 & 0 \\
\hline SS73 24 & $104557.13-595643.1$ & Be pec & 31.70 & -5.65 & 3.13 & 19 \\
Tr 16 64 & $104504.75-594053.7$ & B1.5 Vb & 31.31 & -6.12 & 2.73 & 30 \\
MJ 327 & $104430.34-593726.8$ & B0 V & 31.24 & -7.18 & 1.68 & 0 \\
MJ 427 & $104454.06-594129.4$ & B1 V & 31.10 & -6.51 & 2.94 & 0 \\
MJ 99 & $104343.55-593403.4$ & B2 V & 31.09 & -6.13 & 2.74 & 0 \\
HD 93501 & $104622.02-600118.8$ & B1.5 III: & 31.09 & -6.98 & 6.50 & 14 \\
Coll 228 68 & $104400.17-600607.7$ & B1 Vn & 31.01 & -6.52 & 2.54 & 2 \\
MJ 224 & $104405.84-593511.6$ & B1 V & 31.01 & -6.72 & 1.96 & 12 \\
HD 93190 & $104419.63-591658.6$ & B0 IV:ep & 30.98 & -7.98 & 2.35 & 1 \\
MJ 181 & $104357.96-593353.4$ & B1.5 V & 30.98 & -6.40 & 2.13 & 40 \\
MJ 126 & $104345.04-595325.0$ & B2 V & 30.94 & -6.44 & 1.96 & 0 \\
MJ 289 & $104422.51-593925.4$ & B1.5 V & 30.90 & -6.45 & 0.98 & 0 \\
MJ 184 & $104358.45-593301.5$ & B1 V & 31.07 & -6.54 & 2.38 & 92 \\
MJ 218 & $104405.09-593341.4$ & B1.5 V & 30.76 & -6.77 & 2.49 & 16 \\
\hline
\end{tabular}

Five of the O-type binaries (HD 93403, HD 93205, HD 93129A, HD 93343 and QZ Car) show hard x-rays and high $\log L_{\mathrm{X}} / L_{\mathrm{bol}}>-7.2$, indicating some colliding wind emission. The remaining $\mathrm{O}$ binaries have $-7.2>\log L_{\mathrm{X}} / L_{\mathrm{bol}}>-7.7$, much like many of the $\mathrm{O}$ single stars, confirming a finding of the XMM survey of the Carina region by Antokhin et al. (2008). All of these stars have soft X-ray spectra and show no strong time variability, consistent with the x-ray emission expected from wind shocks embedded in an O-star wind (Owocki \& Cohen 1999). One short-period O5.5 Vz + O9.5 V binary (FO 15) has very low $\log L_{\mathrm{X}} / L_{\mathrm{bol}}=-8.26$. Not only does this star show no evidence of colliding wind shocks, the expected embedded wind shock emission from the O5.5 primary appears to be suppressed by the radiation of the closely orbiting O9.5 secondary.

The X-ray emission for the B stars is more difficult to untangle, partly because their binary status is not known. As a group the early B stars do not follow the $L_{\mathrm{X}} / L_{\mathrm{bol}}$ trend of most $\mathrm{O}$ stars. The most notable of these B stars is a new candidate Herbig Be star, SS73 24, in the Treasure Chest Cluster, with $\log L_{\mathrm{X}}=31.7$ and $\log L_{\mathrm{X}} / L_{\mathrm{bol}} \approx-5.6$. The early B stars that are detected with Chandra often show hard X-rays and higher $L_{\mathrm{X}}$ than expected from embedded wind shocks (see Table 1). Some of these may have unseen, lower-mass PMS coronal companions, but as a group, the Carina B stars detected with Chandra are too X-ray luminous, even if they all harbored $1-3 M_{\odot}$ companions. We emphasize that most of the known B stars in Carina are not detected with Chandra, or are weak X-ray sources. But the X-rays on the most active B stars must be produced by some intrinsic mechanism, possibly related to magnetic fields.

\section{References}

Antokhin, I. I., Rauw, G., Vreux, J.-M., van der Hucht, K. A. et al. 2008, A\& A, 477, 593 Gagné, M., Oksala, M. E., Cohen, D. H., Tonnesen, S. K. et al. 2005, ApJ, 628, 986 Owocki, S. P. \& Cohen, D. H. 1999, ApJ, 520, 833

Stevens, I. R., Blondin, J. M. \& Pollock, A. M. T. 1992, ApJ, 386, 265

ud-Doula, A. \& Owocki, S. P. 2002, ApJ, 576, 413 